\title{
Angle closure glaucoma - misleading causes, delayed treatment
}

\author{
Anca Delia Pantalon*, Crenguța Ioana Feraru, Dorin Chiseliță
}

Ophthalmology Department, „Sf. Spiridon” Emergency Clinical Hospital, „Grigore T. Popa” University

of Medicine and Pharmacy lasi, Romania

\begin{abstract}
We present the clinical case of a 43 years old female patient, referred to our clinic for a red, painful left eye. Multiple bilateral similar attacks were reported by the patient in the last 2 years, for which a diagnosis of conjunctivitis or anterior uveitis was established. At current presentation we found bilateral marked inflammatory reaction in the anterior segment (extensive peripheral iris synechiae, inflammatory membrane in the pupillary area, iris "bombe", pigment dispersion, but no keratic precipitates or cells in the anterior chamber. Intraocular pressure (IOP) was $12 \mathrm{mmHg}$ in OD and $40 \mathrm{mmHg}$ in OS, under topical treatment, started 24 before the current visit. Gonioscopy showed closed angle in both eyes, "openable" in various grades after indentation in all quadrants. Anterior segment ocular coherence tomography (AS-OCT) and ultrasonic biomicroscopy (UBM) suggested anatomical causes for acute angle closure, revealing multiple rolling folds on the iris surface, high insertion onto the scleral wall. Multiple laboratory investigations excluded any potential cause of uveitis, therefore the anatomical theory remained in discussion related to a disproportion between anterior structures leading to angle closure attacks. We performed laser peripheral iridotomy, in this patient with positive outcome: IOP decrease, deepening of the AC, open angle in gonioscopy. Misleading issues in this case confused the initial diagnosis and delayed the adequate treatment.
\end{abstract}

Keywords: angle closure glaucoma, pupillary block, iris crowding, plateau iris syndrome

\section{Introduction}

Primary angle closure is an anatomic disorder. Just as open angle glaucoma is considered a group with diverse etiology connected to a final pathway, angle closure glaucoma needs to be regarded in a similar manner. Angle closure "glaucoma" is not a single entity, but a cluster of clinical entities leading to iris apposition to trabecular meshwork. These diseases are characterized by abnormal relationships between anterior segment structures, which originate from

Received: July 2016; Accepted after review: September 2016; Published: September 2016.

*Corresponding author: Anca Delia Pantalon, PhD student, Ophthalmology Department, "Sf. Spiridon” Emergency Clinical Hospital, „Grigore T. Popa” University of Medicine and Pharmacy,16 Universitatii Str. 700115, Fax: 0232-217781, Tel: +40740686865, lași, Romania.

E-mail: anca pantalon@yahoo.com abnormalities of size or position of structures, which in turn are caused by underlying etiologic factors or initiating events. Apposition of the iris to the trabecular meshwork is the consequence leading to elevated IOP and glaucoma [1].

Primary angle closure can be caused by one or a combination of the following:

- abnormalities in the relative sizes or positions of anterior segment structures;

- abnormalities in the absolute sizes or positions of anterior segment structure;

- abnormal forces in the posterior segment that alter the anatomy of the anterior segment [1].

Regarding secondary causes for glaucoma such as uveitis, IOP raise usually complicates the clinical course of these inflammatory processes, creating good premises to compromise the trabecular drainage either with open or closed angle. Closed angle results through pupillary block 
(posterior synechiae) or forward rotation of the cilliary body without pupillary block [2].

Understanding the anatomic and the pathophysiologic mechanisms involved in the etiology of angle closure glaucoma assists in making the diagnosis and in optimizing the treatment.

\section{Case report}

We present the case of a 43 years old female, admitted in our clinic for severe headaches, photophobia and, a red and painful left eye. Anamnesis revealed similar bilateral episodes that were diagnosed as conjunctivitis or anterior uveitis. All episodes were treated with antibiotics and non-steroidal anti-inflammatory eye drops each time, without thorough investigations over the causes. Last episode in OD occurred 2 months before current presentation, but ocular pain was described by the patient as a new symptom compared to other episodes. No visual acuity decay was ever reported. At that time, an IOP of $43 \mathrm{mmHg}$ was found in OD, for which she received topical medication (bimatoprost and timolol, Ganfort@). Resolution was fast and the medication was withdrawn after 2-3 weeks.

At current presentation visual acuity in $O D$ was $1.0(\mathrm{sc}, \mathrm{nc})$ and $0.8(\mathrm{sc}, \mathrm{nc})$ in OS. IOP $\mathrm{OD}=12 \mathrm{mmHg}$, IOP $\mathrm{OS}=40 \mathrm{mmHg}$, under topical medication (timolol and travoprost, Duotrav ${ }^{\circledR}$ ) started $24 \mathrm{~h}$ before her visit in our service, after an ambulatory consult.

Biomicroscopy of the anterior segment was summarized in Table 1.

Table 1. Slit lamp examination

\begin{tabular}{|c|c|c|}
\hline & OD & OS \\
\hline Conjunctiva & Normal color & Severe congestion \\
\hline Sclera & White, silent eye & Dilated episcleral vessels \\
\hline Cornea & Transparent, normal thickness, no KP & Mild epithelial edema, no KP \\
\hline AC & \multicolumn{2}{|c|}{ Absent in periphery, normal depth in the center; cellular Tyndall $=\varnothing$} \\
\hline Iris & \multicolumn{2}{|c|}{$\begin{array}{l}\text { "Iris bombe", fine IPS/ } 360^{*} \text {; atrophic iris stroma, multiple rolling folds near the iris scleral } \\
\text { insertion, supero-temportal rotation of the entire iris (Figure 1) }\end{array}$} \\
\hline Pupil & \multicolumn{2}{|c|}{ Medium mydriasis, not reflex, pupillary diameter $=4 \mathrm{~mm}$, mild corectopia (supero-temporal) } \\
\hline Lens & \multicolumn{2}{|c|}{ Normally positioned, transparent } \\
\hline $\begin{array}{l}\text { Lacrimal } \\
\text { adnexa }\end{array}$ & Normal & Intense hyperlacrimation \\
\hline
\end{tabular}

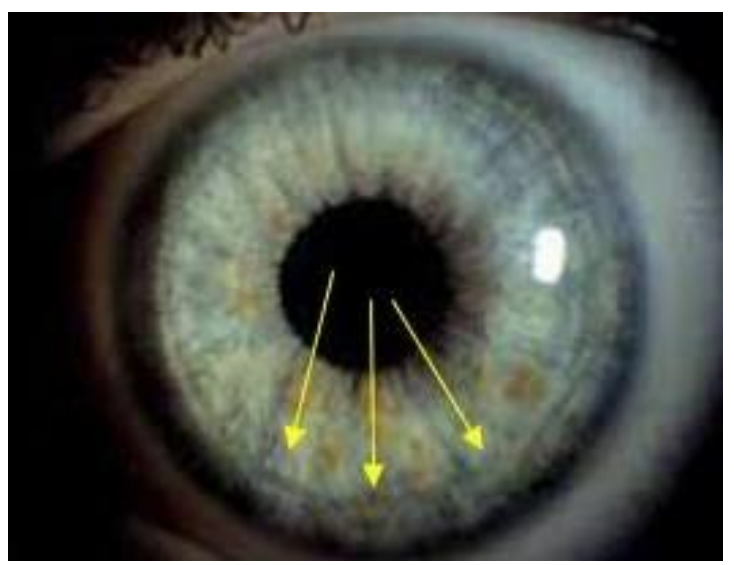

Fig. 1. Slit lamp aspect in OD; no photography was possible in OS due to patient's extreme discomfort during examination.
Gonioscopy revealed closed angles in both eyes; after indentation in OD the angle opened grade III (Schaffer)/ $360^{*}$ and in OS the angle opened grade I-II (Schaffer)/ 360*. Particular iris aspect was noted bilaterally as "double hump" sign, highly suggestive for "plateau iris" configuration or syndrome (well known as anatomical determining factor of acute angle closure/ glaucoma).

Fundus examination was normal in both eyes, except a slight asymmetry in $\mathrm{C} / \mathrm{D}$ ratio (Figures 2 and 3 ). 

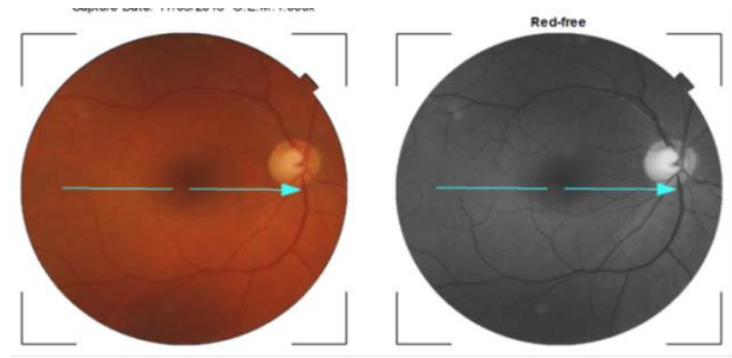

Fig. 2. OD - Normal fundus examination, "red free" included $(C / D$ ratio $=0.4)$

Despite several suggestive elements for secondary inflammatory glaucoma, we also tested various anatomic features of the anterior chamber through ultrasonic biometry (UBM), having in mind that eyes with primary angle closure might have similar inflammatory findings, after recurrent attacks. In these cases significant biometric changes compared to normal eyes are detected (shallower AC, thicker lens, shorter axial lengths and a certain tendency towards hyperopia). [2] Yet if plateau iris syndrome/ configuration is considered, these parameters might be within normal range compared to "normal" configured eyes [2]. Biometry (normal measurements in our patient) was summarized in Table 2. Anterior chamber (AC), although absent in periphery, proved normal depth centrally (important element for primary causes of angle closure and not secondary related to pupillary block in anterior uveitis).

Table 2. Ultrasonic biometric measurements

\begin{tabular}{cc}
\hline OD & OS \\
\hline $\mathrm{K}=43.75 \mathrm{D}$ & $\mathrm{K}=44.40 \mathrm{D}$ \\
$\mathrm{AL}=23.10 \mathrm{~mm}$ & $\mathrm{AL}=23.56 \mathrm{~mm}$ \\
$\mathrm{AC}=2.81 \mathrm{~mm}$ & $\mathrm{AC}=2.81 \mathrm{~mm}$ \\
$\mathrm{~L}=4.09 \mathrm{~mm}$ & $\mathrm{~L}=3.92 \mathrm{~mm}$ \\
$\mathrm{IOL}(118.0)=21 \mathrm{D}$ & $\mathrm{IOL}(118.0)=20 \mathrm{D}$ \\
\hline
\end{tabular}

$\mathrm{K}$ - mean keratometry, $\mathrm{AL}$ - axial lengths, $\mathrm{AC}$-anterior chamber, L - lens thickness, IOL - artificial intraocular lens power calculation

By looking through patient's personal files we found interesting details from previous anterior segment imaging sessions, taken a few months before the current evaluation. Thus, anterior segment coherence tomography (AS-OCT) revealed anteriorly displaced peripheral iris creating the premises of apposition and angle closure. Anterior chamber is moderately deep, the iris and iris
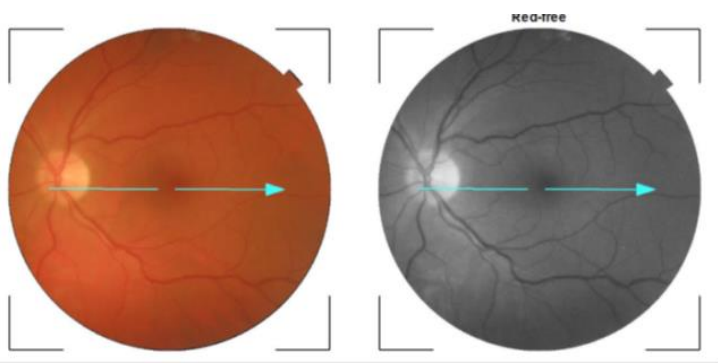

Fig. 3. OS - Normal fundus examination, "red free" included $(C / D$ ratio $=0.2)$

root are comparatively thick and the iris surface is planar. Ciliary processes are also anteriorly positioned and the ciliary sulcus, although present, is minimally defined (Figure 4). Progressive anterior dislocation of the ciliary body with bolstering of the peripheral iris and formation of peripheral anterior synechiae has been described in cases with plateau iris (long term follow up) by ultrasonic biomicroscopy (UBM) [2], as it is the case in our patient (Figure $5 \mathrm{a}, \mathrm{b}$ ).

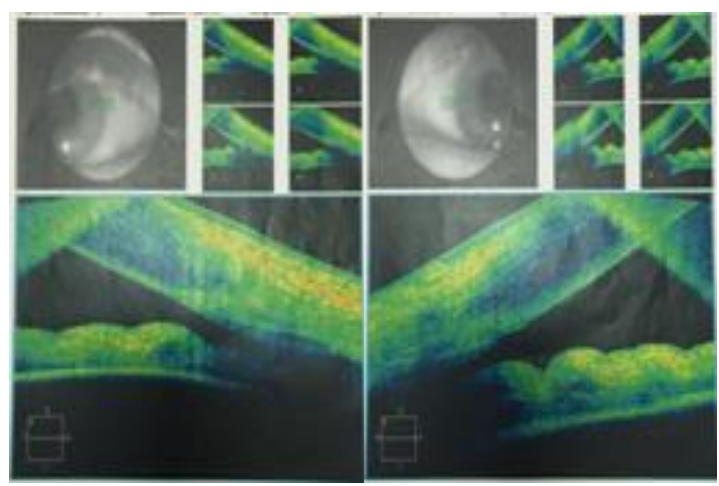

Fig. 4. (OU) Anterior segment OCT aspect narrow angle, prominent angulation of the peripheral iris

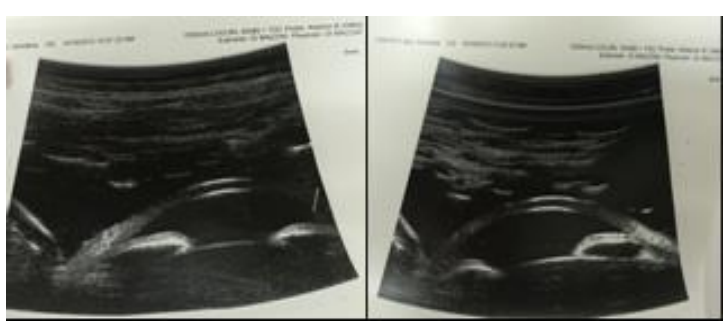

Fig. 5a. OD UBM aspect (centrally deep AC, closed angle, peripheral iris synechiae, flat iris surface and ciliary body touching the posterior iris) (suggestive for plateau iris) 


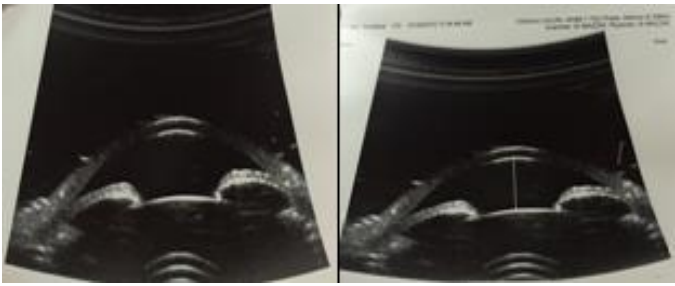

Fig. 5b. OS UBM aspect (centrally deep AC closed angle, peripheral iris synechiae flat iris surface and ciliary body touching the posterior iris)

Immediate treatment was initiated with systemic hyperosmotics (Manitol solution $20 \%$, $250 \mathrm{ml}$, iv), mild diuretics (acetazolamide - 500 mg po) and topical steroids (dexamethasone) 6 times a day in OS. We obtained a significant IOP decrease to: $8 \mathrm{mmHg}$ in OD and to 28 $\mathrm{mmHg}$ in OS. Pilocarpine (2-8\%), although indicated in acute angle closure cases once the pressure decreases below $30 \mathrm{mmHg}$, was avoided in this case due the iris synechias present in the $A C$, as it would have enhanced intraocular inflammation. Peripheral laser iridotomy was performed to release the pupillary block. After the procedure, the AC became overall deeper (Figures 6-8), the angle opened in gonioscopy (Figures 9 and 10) and the IOP decreased to a satisfactory level in OS. Prophylactic iridotomy was also performed in OD to prevent later attacks of angle closure with good result in the postoperatory period.

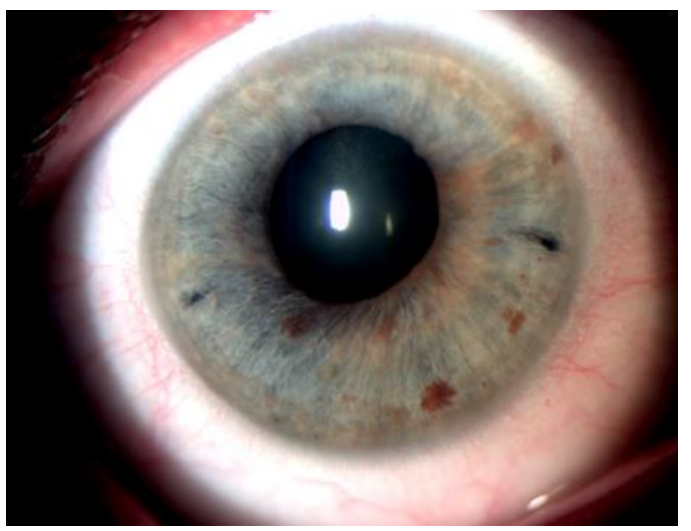

Fig. 6. OD - status post peripheral laser iridotomy $\mathrm{OU}$ - mild corectopia and rotation of iris stroma towards supero-temporal side, mydriasis $O S>O D$, patent $\mathrm{PI}$;

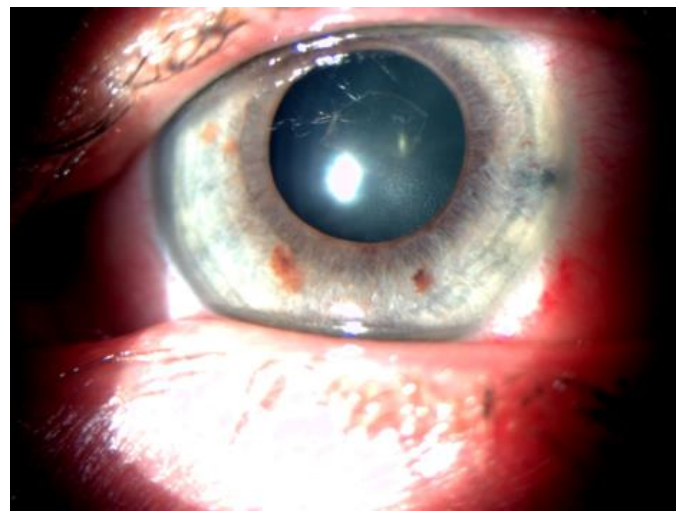

Fig. 7. OS - status post peripheral laser iridotomy (inflammatory membrane visible in the pupillary area)

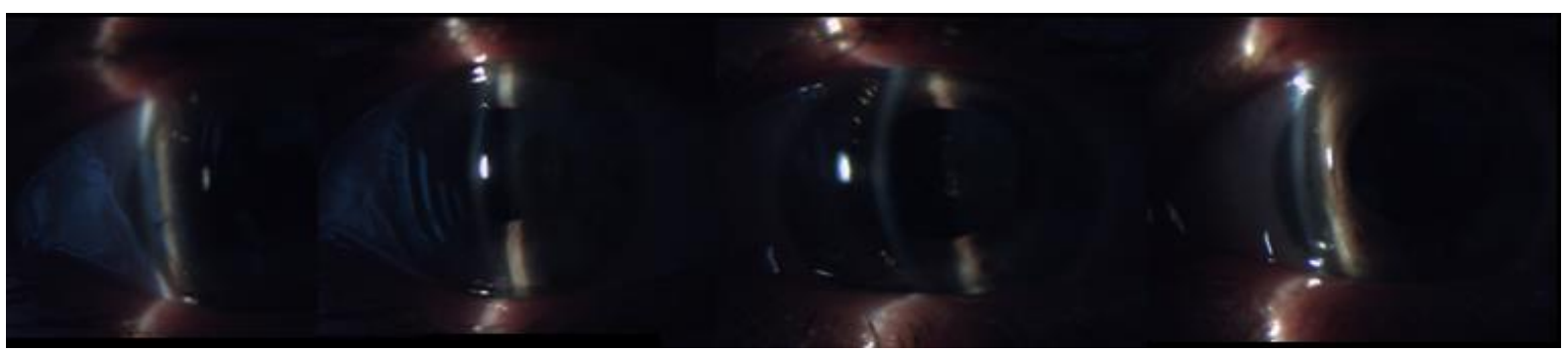

Fig. 8. AC deepened compared to baseline, but still shallower than expected in periphery after the laser treatment; central AC depth was normal (sustainable diagnostic elements for acute angle closure due to plateau iris syndrome/ configuration) 


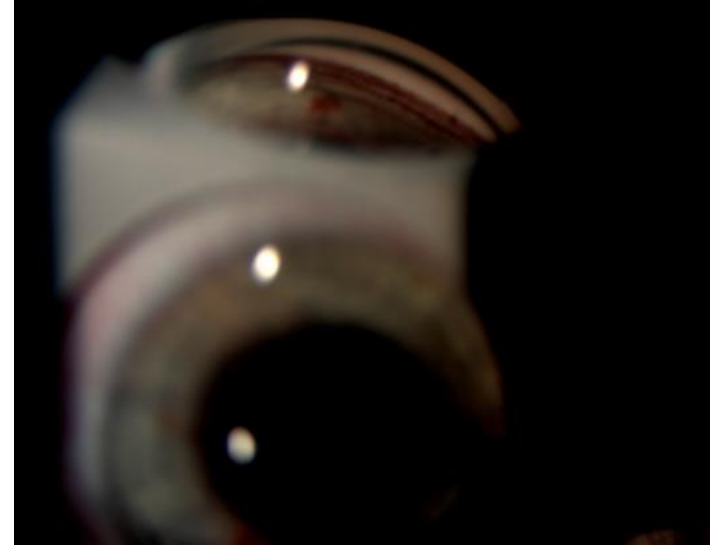

Fig. 9. Gonioscopy aspect OD anterior chamber angle (open/ 360*), grade III Schaffer

Patient received after laser surgery only topical anti-inflammatory medication (steroidal and non-steroidal); for preventing IOP spikes, she was prescribed timolol and dorzolamide (Cosopt®) with good control of IOP in both eyes $(\mathrm{IOP} O D=12 \mathrm{mmHg}$, IOP OS $=10$ $\mathrm{mmHg}$ ). Cosopt was withdrawn in 2 weeks after laser iridotomy, as no further adjunctive lowering IOP medication was needed.

To rule out thoroughly potential causes of ocular inflammation responsible for extensive PAS and angle closure (pupillary block), exhaustive laboratory investigations were performed both during our hospitalization period and in the year prior to her presentation in our service in a uveitis center in Italy. All results were within normal limits or negative, so no sustainable cause for uveitis could be found in our patient (blood cell count, ESR, CRP, fibrinogen, coagulation parameters, blood glucose, creatinine, ALAT, ASAT, cholesterol profile, triglicerides, FTA-Abs, HIV, $\mathrm{HCV}$ Abs, HBs Ag, DNAdc Abs, anti cardiolipine Abs (IgM/lgG), Anti beta2 AbsGPI (lgM/lgG), ANA Abs= $<0.1 \quad(<1 \quad \mathrm{Al})$, cANCA Abs, pANCA Abs, anti Sm Abs, anti Sm RNP, anti RNP 68 Abs, anti SSA/Ro Abs, anti SSB/La Abs, anti SCL-70 Abs, anti Jo1/ RNP A Abs, rheumatoid Factor, HLA B27 panel, ACE).

No pathologic changes were detected either in MRI examination (spinal cord/ sacroiliac joints) or thoracic $X$-ray and corroborated with the lab results most causes for ocular inflammation were excluded.

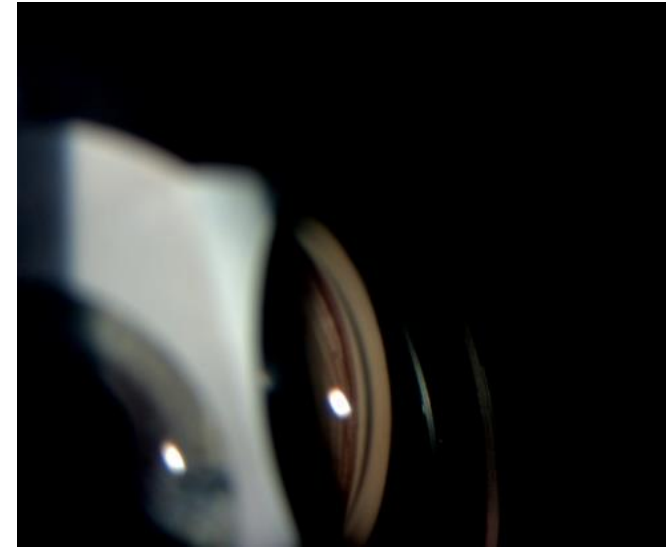

Fig. 10. Goniscopy aspect OS anterior chamber angle (open/ $360^{*}$ ), grade III Schaffer, frequent transverse iris processes

Before release, our patient was investigated and assessed for potential structural and functional glaucoma damage. Therefore we performed a visual field test (Humphrey Field Analyzer, 24-2 SITA Standard strategy, Carl Zeiss Meditech ${ }^{\circledR}$ ) and an optical coherence tomography, to image macular area, optic disk and retinal nerve fiber layer (OCT Topcon®). Normal results were collected at each test, concluding that there was no glaucoma damage in our patient.

From our point of view, close long term follow up became impossible for this patient, as she left the country, but we stayed in contact. As such, we knew that at further visits patient developed increased IOP in OD (2 consecutive measurements with $\mid \mathrm{OP}>21$ $\mathrm{mmHg}$ ), so Cosopt was reintroduced as topical anti glaucomatous medication by her ophthalmologist in Italy. Under this medication IOP in OD was $16 \mathrm{mmHg}$, while in OS laser iridotomy maintained by itself a good IOP control (12 $\mathrm{mmHg}$, without any treatment).

Situation was considered stable for this patient, but regular checkups for angle status assessment (gonioscopy, UBM, AS-OCT) and IOP measurement are necessary for a proper follow up. Moreover this patient needs consistency in following a single opinion because, unless a unanimous opinion is reached, our patient will balance between primary or secondary causes of angle closure glaucoma (anatomic causes vs. uveitic causes). Definitive treatment in this case would be in our opinion ALPI (argon laser peripheral iridoplasty) to "shrink" the iris 
insertion and open the anterior chamber angle, and not topical suppressants of aqueous secretion that treat the effect (increased IOP) and not the underlying cause.

\section{Discussions}

Angle closure glaucoma can present with a spectrum of symptomatology, from none at all to severe pain, blurred vision and nausea. Each patient must be considered a unique case, as presentation may vary in each case. Intermittent angle closure episodes may mimic intraocular inflammation that resolve spontaneously, leaving mild sequels which could mislead the inexperienced ophthalmologist. Because the eyes appear normal between attacks except for a narrow angle, the diagnosis is frequently missed, and the doctors are misled by the patient's selfdiagnosis of migraine, sinusitis, anxiety or eyestrain [1].

The greatest danger lies in the possibility of sudden conversion to acute angle closure glaucoma. Laser iridotomy is definitive if the eye is otherwise normal and the angle not occludable by other mechanisms other than pupillary block. Attacks may occur over months or years, finally leading to an acute attack. Subacute attacks are much more common and tend to produce a chronically dilated pupil, mild iris atrophy, IPS, pigment on the iris and in the anterior chamber (mimicking an anterior uveitis).

In our patient, there were some confusing data that created a delay in establishing the correct diagnosis and treating in consequence the real causes of acute angle closure. Laser treatment cancelled the pupillary block produced by extensive IPS, but as the underlying mechanism was complex, it solved temporarily the problem.

Plateau iris syndrome results from anteriorly positioned ciliary processes holding up the peripheral iris and maintaining its apposition to the trabecular meshwork. Most patients are females, young and less hyperopic than those with pupillary block; often they have a positive family history of angle closure glaucoma. However, because of the nature of the anatomic relationships of the structures surrounding the posterior chamber, the degree of relative pupillary block necessary to induce angle closure is less than that in pure pupillary block angle closure glaucoma [2]. The difference seems to account for the deeper anterior chamber and flatter iris surface in eyes with angle closure and plateau iris. Patients with plateau iris who develop angle closure are also younger than those with pupillary block angle closure glaucoma. As a general rule, the older the patient is, the less prominent the angulation of the peripheral iris and the greater the element of pupillary block. Iridotomy is successful at opening the angle when a component of pupillary block is present, but periodic gonioscopy remains indicated, as the angle can narrow further as it was the case of our patient [3].

In this patient, supplementary to the iris plateau configuration/ syndrome, the UBM evaluation revealed a disproportion between the volume of the iris and the volume of the entire anterior chamber, adding a new contributing element ("iris crowding") to the angle closure mechanism [4].

A recent classification [5] mentions possible mechanisms of angle closure and states all distinctive clinical elements in each group (Figures 14-18). A clear difference between them it very difficult to establish between laser therapies.

I. Angle closure through "pure" pupillary block: : shallow anterior chamber (AC), "iris bombè"; after laser iridotomy the aspect of the iris becomes planar and the angle opens widely (Figure 14);

II. Angle closure without "pupillary block" (iris "crowding"/ anterior ciliary body insertion): deep central AC, peripheral laser iridotomy doesn't change the iris appearance (abrupt peripheral angulation) $\rightarrow$ the angle stays narrow/ closed (Figures 15 and 16);

III. Mixt mechanisms: combined elements (I) + (II): deep central AC, iris "bombè" (Figures 17 and 18); most complex combination was found in our patient (Figure 19). 


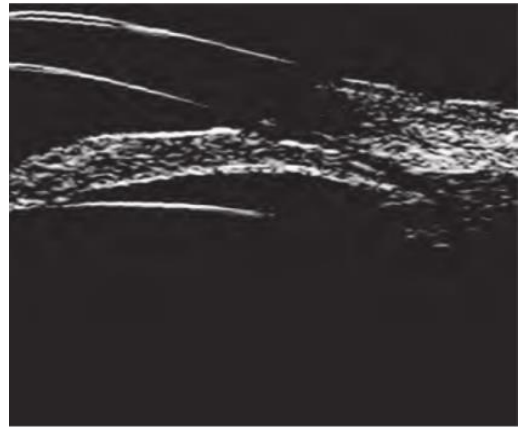

Fig. 14. "Pure" pupillary block (I)

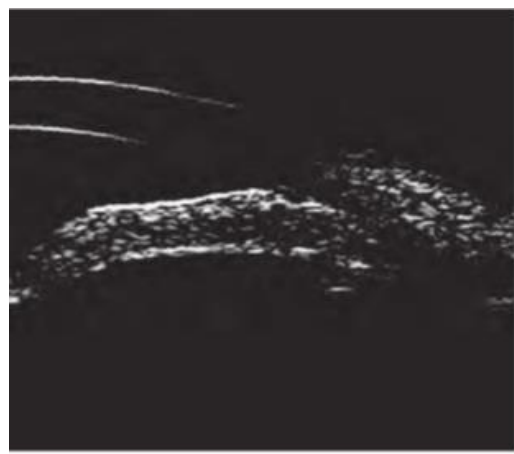

Fig. 17. Pupillary block + "iris crowding" (IIla)

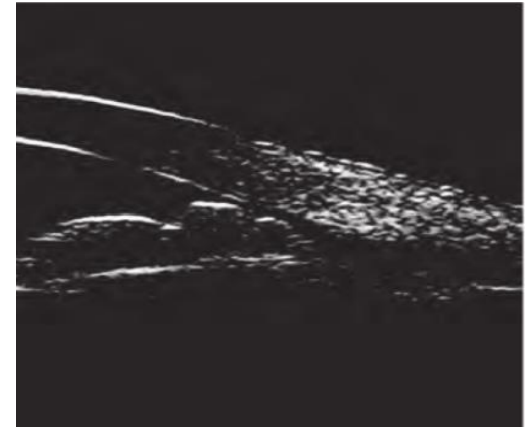

Fig. 15. "Iris crowding" (Ila)

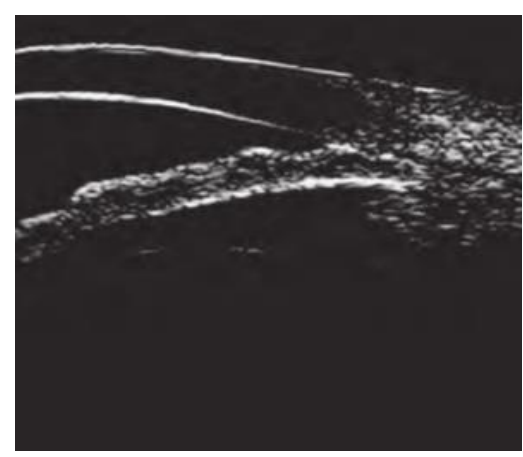

Fig. 18. Pupillary block + anterior insertion of ciliary body (IIllb)

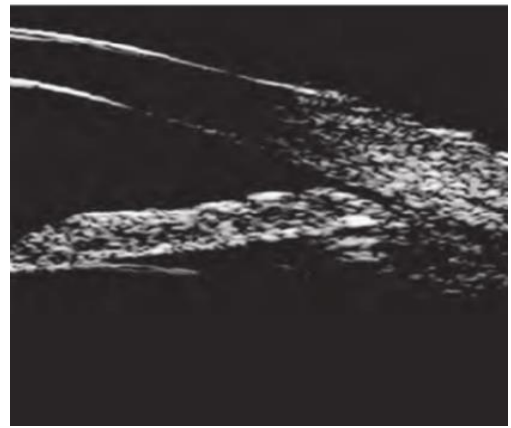

Fig. 16. "Iris crowding" + anterior insertion of ciliary body (Illb)

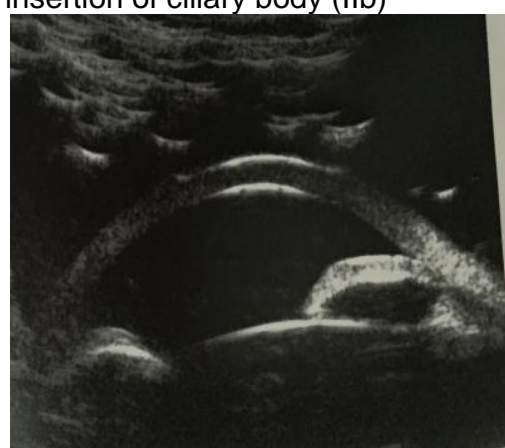

Fig. 19. Pupillary block + "iris crowding" + anterior insertion of ciliary body (our patient!)
Peripheral iridotomy annulled the pure pupillary block compound, put the situation "on hold" for a while but solved the problem only temporary [3]. Adjunctive therapy (Argon laser peripheral iridoplasty - ALPI) not convinced that her problem is anatomical and not inflammatory; therefore it has become difficult to manage the situation. An extremely useful exam would be in this case another UBM and AS-OCT to evaluate exactly the anatomy of the angle $[2,6]$. Since the patient has left the country it has become very difficult for us to manage the situation and to convince the patient what is best to do in her case.

Miotics and ALPI are extremely useful in keeping the AC angle open [3], but are not applicable here since our diagnosis is disregarded and further uveitis cases are considered for investigation. Topical therapy (Cosopt) is efficient, but should not have been chosen as first line of treatment, since cause should be treated first and not the effect [4].

Clear lens extraction might have been or not at option in our patient. Authors tried to follow the European Glaucoma Society Guidelines recommendations [7] that state that in an eye with a clear lens first IP should be performed. If the angle does not open and IOP is not controlled with unquestionable glaucomatous damage, then phacoemulsification and IOL implantation should be considered. Still, clinical reports of phacoemulsification with posterior chamber intraocular lens (IOL) implantation in the treatment of acute, chronic, and secondary angle closure +/- glaucoma describe favorable results.

ALPI is the second step in EGS recommendations in cases with "plateau iris syndrome" as is stretches the iris and widens the chamber angle. It is an useful procedure to eliminate the appositional angle closure resulting from mechanisms other than pupillary block.

Topical treatment and rigorous monitoring (goniscopy included) remains the key in this patient.

\section{Conclusions}

This case shows a confusion of diagnosis that postponed establishing the correct etiology, therefore the treatment. Initial "low grade" inflammatory signs might have been misleading, although some of other signs 
attributable to real anterior uveitis were missing. Our suggestion remains towards establishing a consensus between ophthalmologists to our patient's best interest. The key in this case consists in a correct monitoring strategy in the same ophthalmology center for a proper follow up, treatment and prevention of complications. A long term prognosis in this patient remains difficult to evaluate.

\section{References}

1. Ritch $R$, Shields MB, Krupin $T$. The glaucomas, Volume 1. Philadelphia: Mosby-Elsevier Saunders, 1996: 560-561.

2. Pavlin CJ, Harasiewicz K, Foster FS. Ultrasound biomicroscopy of anterior segment structures in normal and glaucomatous eyes, Am J Ophthalmol 1992; 113(4):381-389.

3. Ritch R, Tham CC, Lam DS. Long-term success of argon laser peripheral iridoplasty in the management of plateau iris syndrome. Ophthalmology 2004; 111(1):104-108.

4. Wright C, Tawfik MA, Waisbourd M, Katz LJ. Primary angle-closure glaucoma: an update. Acta Ophthalmol 2016; 94(3):217-225.

\section{Conflict of interest}

Authors declare no conflicts of interest.

\section{Patient consent}

Written informed consent was obtained from the patient for publication of this case report and accompanying images. A copy of the written consent is available for review by the Editor-in-Chief of this journal.

5. Maslin JS, Barkana Y, Doraira SK. Anterior segment imaging in glaucoma: An updated review. Indian J Ophthalmol 2015; 63(8):630640.

6. Kong $\mathrm{X}$, Foster $\mathrm{PJ}$, Huang $\mathrm{Q}$, et al. Appositional Closure Identified by Ultrasound Biomicroscopy in Population-Based Primary Angle-Closure Glaucoma Suspects: The Liwan Eye Study. Invest Ophthalmol Vis Sci 2011; 52(7):3970-3975.

7. Terminology and Guidelines For Glaucoma 4th Edition by EGS-European Glaucoma Society, Florence, ISBN: 9788898320059. (http://www.eugs.org/eng/EGS_guidelines4.asp \# available at 09/30/2016) 